\title{
Atherosclerotic vascular disease and its correlates in stable black South African kidney transplant recipients
}

This article was published in the following Dove Press journal: International Journal of Nephrology and Renovascular Disease

\author{
Stephen Olawale Oguntola' \\ Muzamil Olamide Hassan ${ }^{2}$ \\ Raquel Duarte ${ }^{3, *}$ \\ Therese Dix-Peek ${ }^{3}$ \\ Caroline Dickens ${ }^{3}$ \\ Gbenga Olorunfemi ${ }^{4}$ \\ Ahmed Vachiat ${ }^{5}$ \\ Graham Paget ${ }^{1}$ \\ Pravin Manga ${ }^{5}$ \\ Saraladevi Naicker ${ }^{1, *}$ \\ 'Department of Internal Medicine, \\ Division of Nephrology, University \\ of Witwatersrand, Johannesburg, \\ South Africa; ${ }^{2}$ Department of Internal \\ Medicine, Dialysis Unit, Ladoke \\ Akintola University of Technology \\ Teaching Hospital, Osogbo, \\ Nigeria; ${ }^{3}$ Department of Internal \\ Medicine Laboratory, University of \\ Witwatersrand, Johannesburg, South \\ Africa; ${ }^{4}$ Department of Epidemiology \\ and Biostatistics, School of Public \\ Health, University of Witwatersrand, \\ Johannesburg, South Africa; \\ ${ }^{5}$ Department of Internal Medicine, \\ Division of Cardiology, University of \\ Witwatersrand, Johannesburg, South \\ Africa
}

*These authors contributed equally to this work

Correspondence: Stephen Olawale Oguntola

Division of Nephrology, Department of Internal Medicine, Faculty of Health Sciences, University of Witwatersrand, 7 York Road, Parktown, Johannesburg,

2193, South Africa

Tel +27 78I489295

Email Olawaleogun I@gmail.com
Background: Despite remarkable improvement in renal function attributable to kidney transplantation, the burden of cardiovascular disease (CVD) among kidney transplant recipients (KTRs) remains high in the post-transplant period. Aggressive use of statins in KTRs may make lipoprotein ratios correlate better with atherosclerotic vascular disease (AsVD) when compared with traditional lipid profile parameters. We therefore evaluated the clinical and echocardiographic correlates of AsVD among non-diabetic, stable, black KTRs in South Africa. Methods: This was a cross-sectional study of 41 adult (18-65 years), non-diabetic, stable KTRs and 41 age- and sex-matched healthy controls. An interviewer-administered questionnaire was used to obtain information on participants' sociodemographic and cardiovascular risk factors. Anthropometric parameters were measured. Urine and blood samples were obtained and analyzed. Echocardiography was performed and carotid intima media thickness (CIMT) was assessed in both right and left carotid arteries. Spearman's rank correlation and binary logistic regression were performed to determine the relationship between CVD risk factors and AsVD. Results: AsVD was present in $46.3 \%$ of KTRs compared to $17.1 \%$ of healthy controls $(p=0.004)$. Left ventricular hypertrophy was present in $92.7 \%$ of the KTRs. There were statistically significant differences in waist-hip ratio, systolic blood pressure, mean arterial pressure, urine albumin-creatinine ratio, serum fibrinogen, serum creatinine, estimated glomerular filtration rate, left atrial diameter, left ventricular mass (LVM), and left ventricular mass index (LVMI) between KTRs and controls. A positive relationship was seen between CIMT and certain risk factors for CVD including LVM, LVMI, and mitral valve deceleration time, $(p<0.001)$. Castelli index 2 and lipoprotein combine index (LCI) showed positive correlation with CIMT. On multivariate analysis, increasing age and kidney transplant status were independent predictors of AsVD after controlling for other risk factors.

Conclusion: AsVD was common among KTRs. Older age and kidney transplant status independently predicted AsVD. Castelli index 2 and LCI correlated with AsVD better than serum lipid parameters.

Keywords: carotid intima media thickness, lipoprotein ratios, Castelli index 2, lipoprotein combine index, left ventricular mass index

\section{Introduction}

Kidney transplantation offers a greater survival benefit compared to dialytic therapy in end-stage kidney disease (ESKD) patients; ${ }^{1}$ however, a high prevalence of cardiovascular disease (CVD) among kidney transplant recipients (KTRs) predisposes them to increased mortality in comparison to healthy controls. ${ }^{2,3}$ Risk factors for vascular disease identified among KTRs in an American study included age, sex, cigarette smoking, 
pre-transplant splenectomy, and serum albumin. ${ }^{4}$ Traditionally, a favorable lipid profile among black Africans was identified as the reason for a lower prevalence of atherosclerotic vascular disease (AsVD) and ischemic heart disease, despite the presence of other cardiovascular risk factors. ${ }^{5}$ However, a rise has been reported in the prevalence of dyslipidemia and ischemic heart disease in the urban black population of South Africa. ${ }^{6,7}$ This trend has been attributed to increasing urbanization, changes in diet, and a reduction in physical activity. ${ }^{8}$ Additionally, the high prevalence of both traditional and chronic kidney disease (CKD)-related cardiovascular risk factors among ESKD patients has contributed to the high risk of AsVD among this group of patients. A case-control study among black South African ESKD patients reported a higher prevalence of carotid plaques among ESKD patients on maintenance hemodialysis (38.1\%) when compared with controls (7.9\%). ${ }^{9}$ Furthermore, Muhammad et al ${ }^{10}$ found left ventricular hypertrophy (LVH) to be prevalent among KTRs and also reported that a longer duration on dialysis, cigarette smoking, higher cumulative steroid dose, increased carotid intima media thickness (CIMT), and increased waist circumference predicted the presence of LVH. In view of the aggressive use of lipid-lowering medications in CKD and post-kidney transplantation, use of conventional lipid profiles for cardiovascular risk assessment in this group of patients may not provide the whole picture. Lipoprotein ratios may correlate better with AsVD than lipid profile parameters. The discriminatory and predictive power of total cholesterol (TC)/ high-density lipoprotein (HDL) was found to be superior to either TC or HDL. ${ }^{11}$ Lipoprotein ratios have been shown to be superior to conventional lipid profiles in predicting coronary heart disease. ${ }^{12}$ Furthermore, sociodemographic characteristics and possibly genetic variations may impact the cardiovascular risk among KTRs in our environment. Although some studies have evaluated CVD among black ESKD, maintenance dialysis, and renal transplant patients, ${ }^{9,10}$ a significant knowledge gap of the risk factors for AsVD among stable, black KTRs in the absence of diabetes and inflammatory conditions still exists.

In view of the paucity of knowledge about AsVD among black South African KTRs, we evaluated the relationship of dyslipidemia and lipoprotein ratios to AsVD among nondiabetic, stable, black KTRs.

\section{Methods}

This was a comparative cross-sectional study of 41 adult (age 18-65 years) non-diabetic, stable KTRs and 41 age- and sexmatched healthy controls at a large urban public hospital in
South Africa from January 2, 2017 to August 31, 2017. The study was approved by the Human Research Ethics Committee, University of Witwatersrand, Johannesburg, South Africa (Study number 160614). All participants provided signed informed consent before enrollment in the study. An interviewer-administered questionnaire was used to obtain information on the participants' sociodemographic and cardiovascular risk factors.

Waist and hip circumferences were measured with patients in an erect position and waist-hip ratios (WHR) calculated. Body mass index (BMI) was calculated using the formula mass/height ${ }^{2}$, while body surface area was calculated using the Mosteller formula. ${ }^{13}$ Serum fibrinogen was determined using STA-R Max (Stago, Asnières-sur-Seine, France). A serum lipogram (TC, low-density lipoprotein [LDL], triglyceride [TG], HDL) was determined by an enzymatic colorimetric method using the Cobas 8000 modular analyzer series, module c701 analyzer (Hoffman-La Roche Ltd., Basel, Switzerland). Lipoprotein ratios were calculated as follows: atherogenic index of plasma $=\log (\mathrm{TG} / \mathrm{HDL})$; Castelli index 1 = TC/HDL; Castelli index $2=\mathrm{LDL} / \mathrm{HDL}$; non-HDL = TC-HDL; atherogenic index $(\mathrm{AI})=$ non-HDL/ $\mathrm{HDL}$; lipoprotein combine index $(\mathrm{LCI})=(\mathrm{TC} \times \mathrm{TG} \times \mathrm{LDL}) /$ HDL. ${ }^{14}$ The urine albumin-creatinine ratio was determined by an immunoturbidimetric assay, using a Tina-quint Albumin Gen.2 serum/plasma application kit, on the Cobas 8000 modular analyzer, module c502. Estimated glomerular filtration rate (eGFR) was calculated using the 2009 CKDEPI formula. ${ }^{15}$ All participants had echocardiography and CIMT measurements in accordance with the guidelines of the American Society of Echocardiography, ${ }^{16}$ using a Philips iE33 echocardiography machine (Philips, Amsterdam, the Netherlands). CIMT was assessed using the vascular probe of the echocardiography machine, Philips IE33 (S5-1 probe) by focusing on the far wall of the common carotid artery, 1 $\mathrm{cm}$ proximal to the dilatation of the carotid bulb along the long axis of the artery. Automatic echo-generated measurements with percentage quality of $95 \%$ were recorded. The procedure was performed on both the left and right carotid arteries and the average used in analysis.

\section{Data analysis}

Stata version13.1 (StataCorp LP, College Station, TX, USA) was used for statistical analysis. Categorical variables were expressed as frequencies and percentages and compared using the chi-square test. A Shapiro-Wilk test of normality was performed on all continuous variables and normally distributed data were presented as mean $\pm \mathrm{SD}$, while 
non-normally distributed data were presented as median and interquartile range (IQR). Comparisons were performed between KTRs and controls, and between participants who had AsVD and those who did not, using the Student's $t$-test for normally distributed data and the Wilcoxon rank-sum test for non-normally distributed data.

Spearman's correlation was used to determine the relationship between CIMT and cardiovascular risk factors among KTRs and controls. Multivariate regression analysis was performed to determine the relationship and contribution of cardiovascular risk factors to AsVD. Significance was taken as $p<0.05$. Post-regression analysis was performed to determine the goodness-of-fit of the final model.

\section{Results}

The median age was 39 years (IQR: 30-52) among KTRs while the median age among the control group was 41 years (IQR: 29-48), as shown in Table 1. The most common causes of ESKD in the KTR population were hypertension-attributed CKD and glomerulonephritis (both $\mathrm{n}=19$, 46.3\%).

Table I Sociodemographic and clinical characteristics of the study population

\begin{tabular}{|c|c|c|}
\hline Parameter & $\begin{array}{l}\text { KTR } \\
(n=4 I)(\%)\end{array}$ & $\begin{array}{l}\text { Control } \\
(n=4 I)(\%)\end{array}$ \\
\hline Age (years) & $39(30-52)^{*}$ & $41(29-48) *$ \\
\hline \multicolumn{3}{|l|}{ Sex } \\
\hline Female & $20(49.0)$ & $23(56.0)$ \\
\hline Male & $21(51.0)$ & $18(44.0)$ \\
\hline \multicolumn{3}{|l|}{ Marital status } \\
\hline Married & $23(56.0)$ & $30(73.0)$ \\
\hline Single & $18(44.0)$ & II (27.0) \\
\hline \multicolumn{3}{|l|}{ Cause of ESKD } \\
\hline Hypertension & $19(46.3)$ & \\
\hline Glomerulonephritis & $19(46.3)$ & \\
\hline Primary oxalosis & $\mathrm{I}(2.4)$ & \\
\hline Dysplastic kidneys & $2(4.9)$ & \\
\hline \multicolumn{3}{|l|}{ Donor source } \\
\hline Deceased & $38(92.7)$ & \\
\hline RLD & $3(7.3)$ & \\
\hline \multicolumn{3}{|l|}{$\mathrm{CNI}$} \\
\hline Tacrolimus & $37(90.2)$ & \\
\hline Cyclosporine & $4(9.8)$ & \\
\hline Pre-transplant dialysis duration (years) & $5.0(4.0-6.0)^{*}$ & \\
\hline Post-transplant duration (years) & $4.0(1.0-7.0)^{*}$ & \\
\hline \multicolumn{3}{|l|}{ Proteinuria } \\
\hline$\leq 3 \mathrm{mg} / \mathrm{mmol}$ & $19(46.3)$ & $39(95.1)$ \\
\hline$>3 \mathrm{mg} / \mathrm{mmol}$ & $22(53.7)$ & $2(4.9)$ \\
\hline \multicolumn{3}{|l|}{ Hypertension } \\
\hline Absent & $16(39.0)$ & $34(82.9)$ \\
\hline Present & $25(61.0)$ & $7(17.1)$ \\
\hline
\end{tabular}

Note: *Median (interquartile range).

Abbreviations: KTR, kidney transplant recipient; ESKD, end-stage kidney disease; $\mathrm{RLD}$, related living donor; CNI, calcineurin inhibitor.
All the KTRs were on calcineurin inhibitor (CNI)-based immunosuppressive therapy (with more than $90 \%$ on Tacrolimus while $<10 \%$ were on cyclosporine), antimetabolites (30 [73.2\%] were on mycophenolate mofetil, five [12.2\%] were on mycophenolic acid, four [9.8\%] were on azathioprine, and two [4.9\%] were on Leflunomide), and $5 \mathrm{mg}$ maintenance prednisone daily. The majority $(n=38 / 41,92.7 \%)$ of the KTRs had received deceased donor organs while three $(7.3 \%)$ had related living donor transplantation. The median post-transplant follow-up duration was 4 years (IQR: 1-7) while median pre-transplant dialysis duration was 5 years (IQR: 4-6). LVH was present in $34(82.9 \%)$ of the KTRs; $15 / 17(88.2 \%)$ of KTRs with GFR $<60 \mathrm{~mL} / \mathrm{min} / 173 \mathrm{~m}^{2}$ and in $13 / 18(72.2 \%)$ KTRs with elevated blood pressure. Among the KTRs, 24 (58.5\%) had concentric hypertrophy (relative wall thickness [RWT] $>0.42$ and left ventricular mass index [LVMI] $>95 \mathrm{~g} / \mathrm{m}^{2}$ for females or $>115 \mathrm{~g} / \mathrm{m}^{2}$ for males), ten (24.4\%) had eccentric hypertrophy (RWT $\leq 0.42$ and LVMI $>95 \mathrm{~g} / \mathrm{m}^{2}$ for females or $>115 \mathrm{~g} / \mathrm{m}^{2}$ for males), five $(12.2 \%)$ had concentric remodeling (RWT $>0.42$ and LVMI $\leq 95 \mathrm{~g} / \mathrm{m}^{2}$ for females or $\leq 115 \mathrm{~g} / \mathrm{m}^{2}$ for males), and two $(4.9 \%)$ had normal geometry. Using the combination of increased CIMT values $(>0.55 \mathrm{~mm})$ and the presence of plaques, AsVD was present in $19(46.3 \%)$ KTRs compared to seven $(17.1 \%)$ healthy controls, $(p=0.004)$. CIMT was significantly increased among the KTRs compared to the controls ( $p=0.021$ ). As shown in Table 2, significant differences were seen between other echocardiographic measurements when KTRs were compared to controls. There was no association between CNI agents and AsVD, $\left(\chi^{2}=1.46, p=0.321\right)$.

There was a statistically significant difference in Castelli 1 and 2 indices, AI, non-HDL, and LCI in those with AsVD compared to those without AsVD (Table 3). There were no statistically significant differences in the serum levels of TC, TG, LDL, and HDL. Spearman's correlation between CIMT and risk factors for CVD among KTRs revealed a positive relationship with $\operatorname{LVM}(r=0.52, p<0.001)$, LVH $(r=0.53, p<0.001)$, LAD $(r=0.43, p=0.007)$, age $(r=0.43, p=0.005)$, and WHR $(r=0.39, p=0.012)$ as shown in Table 4. Of the lipid profile parameters and lipoprotein ratios in KTRs, only Castelli 2 index and LCI showed correlation with CIMT.

The multivariable model of the predictors of AsVD showed that KTR status confers an 11-fold risk of developing AsVD ( $\mathrm{OR}=11.22,95 \% \mathrm{CI}=1.82-68.93, p=0.009)$; Table 5. The odds of developing AsVD was 17 times higher in subjects $\geq 40$ years $(p=0.001)$. Age and KTR status were independent predictors of AsVD after correcting for WHR, 
Table 2 Comparison of cardiovascular risk factors between kidney transplant recipients and controls

\begin{tabular}{|c|c|c|c|}
\hline Parameters & KTR $(n=4 I)$ & Control $(n=4 I)$ & $p$-value \\
\hline Age (years) & $39(30-52)$ & $41(29-48)$ & $0.824 *$ \\
\hline BMI $\left(\mathrm{kg} / \mathrm{m}^{2}\right)$ & $25.7(23.1-28.5)$ & $28.7(22.9-31.2)$ & $0.08 I^{*}$ \\
\hline WHR & $0.89 \pm 0.07$ & $0.85 \pm 0.06$ & $0.002^{\#}$ \\
\hline $\mathrm{SBP}(\mathrm{mmHg})$ & $139.7 \pm 15.8$ & $125.4 \pm 1 \mid .3$ & $<0.00 I^{\#}$ \\
\hline MAP (mmHg) & $106.9 \pm 13.8$ & $94.6 \pm 11.4$ & $<0.00 I^{\#}$ \\
\hline $\mathrm{TC}(\mathrm{mmol} / \mathrm{L})$ & $4.46(3.93-4.93)$ & $4.27(3.7 \mid-4.70)$ & $0.330 *$ \\
\hline $\mathrm{LDL}(\mathrm{mmol} / \mathrm{L})$ & $2.63(2.15-3.08)$ & $2.59(2.08-3.17)$ & $0.777^{*}$ \\
\hline TG (mmol/L) & $1.31(1.06-1.92)$ & $1.25(0.80-1.53)$ & $0.399 *$ \\
\hline $\mathrm{HDL}(\mathrm{mmol} / \mathrm{L})$ & $1.29(1.05-1.47)$ & $1.15(0.98-1.54)$ & $0.467^{*}$ \\
\hline Urine-ACR (mg/mmol) & $4.60(1.20-16.2)$ & $0.40(0.20-0.80)$ & $<0.001 *$ \\
\hline Fibrinogen $(g / L)$ & $3.00(2.60-3.5)$ & $2.50(2.20-3.20)$ & $0.027^{*}$ \\
\hline $\mathrm{SCr}(\mu \mathrm{mol} / \mathrm{L})$ & $123(9|-| 52)$ & 80 (63-89) & $<0.001 *$ \\
\hline eGFR $\left(\mathrm{mL} / \mathrm{min} / \mathrm{I} .73 \mathrm{~m}^{2}\right)$ & 71 (49-85) & $113(99-124)$ & $<0.00 I^{*}$ \\
\hline $\operatorname{LAD}(\mathrm{mm})$ & $35.9(32.3-34.6)$ & $31.02(29.0-32.2)$ & $<0.00 I^{\#}$ \\
\hline CIMT (mm) & $0.60(0.5 \mathrm{I}-0.66)$ & $0.53(0.47-0.60)$ & $0.021^{*}$ \\
\hline $\operatorname{LVM}(\mathrm{g})$ & $219(187-284)$ & $174(142-226)$ & $0.001 *$ \\
\hline LVMI $\left(\mathrm{g} / \mathrm{m}^{2}\right)$ & $130.0(108.4-165.2)$ & I0I.I (73.8-II5.7) & $<0.001 *$ \\
\hline
\end{tabular}

Notes: \#Mean \pm SD, Student's t-test; *median (interquartile range), Wilcoxon rank-sum test.

Abbreviations: KTR, kidney transplant recipient; BMI, body mass index; WHR, waist-hip ratios; SBP, systolic blood pressure; MAP, mean arterial pressure; TC, total cholesterol; LDL, low-density lipoprotein; TG, triglyceride; HDL, high-density lipoprotein; urine-ACR, urine albumin-creatinine ratio; SCr, serum creatinine; eGFR, estimated glomerular filtration rate; LAD, left atrial diameter; CIMT, carotid intima media thickness; LVM, left ventricular mass; LVMI, left ventricular mass index.

Table 3 Comparison between participants with atherosclerotic vascular disease and without it

\begin{tabular}{|c|c|c|c|c|c|c|}
\hline \multirow[t]{2}{*}{ Parameter } & \multicolumn{2}{|c|}{ KTRs and controls $(n=82)$} & \multirow[t]{2}{*}{$p$-value } & \multicolumn{2}{|l|}{ KTRs $(n=4 I)$} & \multirow[t]{2}{*}{$p$-value } \\
\hline & $\begin{array}{l}\text { AsVD present } \\
(\mathrm{n}=26)\end{array}$ & $\begin{array}{l}\text { ASVD absent } \\
(n=56)\end{array}$ & & $\begin{array}{l}\text { AsVD present } \\
(n=19)\end{array}$ & $\begin{array}{l}\text { AsVD absent } \\
(n=22)\end{array}$ & \\
\hline Age (years) & $48.0(41.0-55.0)$ & $32.5(27.0-44.0)$ & $<0.00 I^{*}$ & $48.0(39.0-62.0)$ & $30.5(26.0-40.0)$ & $<0.001$ \\
\hline WHR & $0.91(0.86-0.93)$ & $0.86(0.83-0.90)$ & $0.028^{\#}$ & $0.91(0.88-0.96)$ & $0.87(0.84-0.90)$ & 0.069 \\
\hline BMI $\left(\mathrm{kg} / \mathrm{m}^{2}\right)$ & $26.5(24.0-31.2)$ & $27.1(21.5-30.0)$ & $0.273 *$ & $26.2(23.7-28.2)$ & $25.3(20.7-28.7)$ & 0.513 \\
\hline $\mathrm{SBP}(\mathrm{mmHg})$ & $139.2(132.6-145.9)$ & I40.| (|32.3-147.0) & $0.855^{\#}$ & $138.0(|28.0-| 47.0)$ & $141.1(\mid 26.0-151)$ & 0.794 \\
\hline AIP & $0.03(-0.03-0.11)$ & $0.02(-0.08-0.12)$ & $0.729 *$ & $0.02(-0.13-0.20)$ & $0.01(-0.15-0.22)$ & 0.917 \\
\hline Castelli I & $4.1(3.1-4.9)$ & $3.1(2.5-4.1)$ & $0.033^{*}$ & $3.53(3.00-4.80)$ & $3.36(2.57-4.16)$ & 0.403 \\
\hline Castelli 2 & $2.5(1.6-3.2)$ & $1.8(1.3-2.6)$ & $0.048 *$ & $2.09(1.53-2.73)$ & $1.67(1.12-2.55)$ & 0.158 \\
\hline $\mathrm{LCl}$ & $15.4(10.1-18.9)$ & $8.7(4.4-19.6)$ & $0.013^{*}$ & $13.38(9.13-16.54)$ & $8.65(4.6 \mathrm{I}-24.32)$ & 0.308 \\
\hline Non-HDL & $3.4(2.8-4.0)$ & $2.9(2.3-3.5)$ & $0.019 *$ & $3.31(2.76-3.77)$ & $3.03(2.36-3.76)$ & 0.381 \\
\hline $\mathrm{Al}$ & $3.0(2.1-3.9)$ & $2.1(1.5-3.2)$ & $0.034 *$ & $2.53(2.00-3.80)$ & $2.36(1.57-3.36)$ & 0.403 \\
\hline $\mathrm{TC}(\mathrm{mmol} / \mathrm{L})$ & $4.5(4 . I-5 . I)$ & $4.3(3.5-4.8)$ & $0.07 I^{*}$ & $4.46(4.14-4.93)$ & $4.47(3.46-5.09)$ & 0.666 \\
\hline $\mathrm{TG}(\mathrm{mmol} / \mathrm{L})$ & $1.3(1.1-2.1)$ & $1.3(0.8-1.7)$ & $0.139 *$ & $1.29(0.99-1.89)$ & $1.32(1.12-1.95)$ & 0.855 \\
\hline $\mathrm{LDL}(\mathrm{mmol} / \mathrm{L})$ & $2.7(2.2-3.3)$ & $2.6(2.0-2.9)$ & $0.087^{*}$ & $2.65(2.19-3.24)$ & $2.49(1.45-2.73)$ & 0.205 \\
\hline $\mathrm{HDL}(\mathrm{mmol} / \mathrm{L})$ & I.I (0.9-I.4) & $1.3(1.1-1.6)$ & $0.163 *$ & $1.16(1.01-1.60)$ & $1.32(1.09-1.47)$ & 0.456 \\
\hline Fibrinogen $(g / L)$ & $3.0(2.6-3.5)$ & $2.5(2.2-3.2)$ & $0.04 I^{*}$ & $3.00(2.70-3.60)$ & $2.95(2.50-3.30)$ & 0.582 \\
\hline Urine-ACR (mg/mmol) & $2.3(0.8-8.1)$ & $0.8(0.3-2.6)$ & $0.056 *$ & $2.60(1.40-10.7)$ & $9.65(1.20-30.8)$ & 0.296 \\
\hline eGFR $\left(\mathrm{mL} / \mathrm{min} / \mathrm{I} .73 \mathrm{~m}^{2}\right)$ & $86.5(54.0-117)$ & $97.5(70.5-116.0)$ & $0.386 *$ & $71.0(44.0-115.0)$ & $70.0(49.0-84.0)$ & 0.497 \\
\hline LAD (mm) & $37.5(32.0-40.0)$ & $32.0(29.0-35.0)$ & $<0.00 I^{\#}$ & $38.0(35.0-41.0)$ & $34.5(30.0-36.0)$ & 0.011 \\
\hline LVMI $\left(g / \mathrm{m}^{2}\right)$ & |35.| (1 |8.2-168.2) & $103.9(81.6-123.5)$ & $<0.00 I^{*}$ & $141.2(|28.2-| 78.0)$ & $116.2(100.7-131.8)$ & $<0.001$ \\
\hline Dec. time (ms) & I 72.5 (|55.0-201.0) & 148.0 (|29.5-|7|.0) & $<0.00 I^{*}$ & $176.0(158.0-204.0)$ & $135.5(\mid 20.0-175.0)$ & 0.003 \\
\hline $\mathrm{EF}(\%)$ & $73.0(64.0-79.0)$ & $68.0(62.5-74.0)$ & $0.229 *$ & $76.0(64.0-80.0)$ & $69.0(64.0-79.0)$ & 0.574 \\
\hline
\end{tabular}

Notes: \#Mean \pm SD, Student's $t$-test; *median (interquartile range), Wilcoxon rank-sum test.

Abbreviations: KTR, kidney transplant recipient; AsVD, atherosclerotic vascular disease; WHR, waist-hip ratios; BMI, body mass index; SBP, systolic blood pressure; AIP, atherogenic index of plasma; LCl, lipoprotein combine index; HDL, high-density lipoprotein; Al, atherogenic index; TC, total cholesterol; TG, triglyceride; LDL, low-density lipoprotein; urine-ACR, urine albumin-creatinine test; eGFR, estimated glomerular filtration rate; LAD, left atrial diameter; LVMI, left ventricular mass index; Dec. time, mitral valve deceleration time; $E F$, ejection fraction.

proteinuria, GFR, Castelli index 2, and LVH. The goodnessof-fit of the model was assessed by the Hosmer-Lemeshow test $(p=0.319)$. Binary logistic regression analysis among
KTRs showed that age $>40$ years independently predicted AsVD after adjusting for post-transplant follow-up duration, duration of dialysis and LVH, Table 6. 
Table 4 Correlation of carotid intima media thickness to risk factors for cardiovascular disease among kidney transplant recipients

\begin{tabular}{|c|c|c|c|c|}
\hline \multirow[t]{2}{*}{ Parameter } & \multicolumn{2}{|c|}{$\operatorname{KTR}(n=4 I)$} & \multicolumn{2}{|c|}{ Controls $(n=4 I)$} \\
\hline & rho & $p$-value & rho & p-value \\
\hline Age (years) & 0.42 & $0.006 *$ & 0.24 & 0.138 \\
\hline WHR & 0.39 & $0.013 *$ & -0.13 & 0.431 \\
\hline BMI $\left(\mathrm{kg} / \mathrm{m}^{2}\right)$ & 0.17 & 0.296 & 0.11 & 0.512 \\
\hline eGFR $\left(\mathrm{mL} / \mathrm{min} / \mathrm{l} .73 \mathrm{~m}^{2}\right)$ & 0.29 & $0.07 I$ & -0.08 & 0.617 \\
\hline $\mathrm{TC}(\mathrm{mmol} / \mathrm{L})$ & 0.20 & 0.203 & 0.19 & 0.230 \\
\hline LDL (mmol/L) & 0.30 & 0.050 & 0.08 & 0.619 \\
\hline $\mathrm{TG}(\mathrm{mmol} / \mathrm{L})$ & 0.09 & 0.566 & -0.02 & 0.919 \\
\hline Castelli I & 0.27 & 0.082 & 0.06 & 0.710 \\
\hline Castelli 2 & 0.33 & $0.035^{*}$ & 0.03 & 0.845 \\
\hline AIP & 0.13 & 0.408 & -0.03 & 0.834 \\
\hline Non-HDL & 0.29 & $0.07 I$ & 0.12 & 0.465 \\
\hline Atherogenic index & 0.28 & 0.082 & 0.06 & 0.710 \\
\hline $\mathrm{LCl}$ & 0.31 & $0.047^{*}$ & 0.04 & 0.813 \\
\hline $\mathrm{EF}(\%)$ & 0.32 & $0.044^{*}$ & 0.06 & 0.710 \\
\hline FS (\%) & 0.27 & 0.090 & 0.02 & 0.884 \\
\hline $\mathrm{E} / \mathrm{e}^{\prime}$ & 0.21 & 0.180 & 0.28 & 0.079 \\
\hline Dec. time $(\mathrm{ms})$ & 0.59 & $<0.00 I^{*}$ & 0.15 & $0.34 I$ \\
\hline LVM (g) & 0.50 & $<0.00 I^{*}$ & 0.24 & 0.132 \\
\hline LVMI $\left(\mathrm{g} / \mathrm{m}^{2}\right)$ & 0.50 & $<0.00 I^{*}$ & 0.25 & 0.111 \\
\hline LAD (mm) & 0.42 & $0.007^{*}$ & 0.14 & 0.373 \\
\hline
\end{tabular}

Note: *Statistically significant, $p<0.05$.

Abbreviations: E/e', ratio of transmitral doppler early filling velocity to tissue doppler early diastolic mitral annular velocity; KTR, kidney transplant recipient; WHR, waist-hip ratios; BMI, body mass index; eGFR, estimated glomerular filtration rate; TC, total cholesterol; LDL, low-density lipoprotein; TG, triglyceride; AIP, atherogenic index of plasma; HDL, high-density lipoprotein; LCI, lipoprotein combine index; EF, ejection fraction; FS, fractional shortening; Dec. time, mitral valve deceleration time; LVM, left ventricular mass; LVMI, left ventricular mass index; LAD, left atrial diameter.

Table 5 Logistic regression showing relationship of atherosclerotic vascular disease to risk factors of cardiovascular disease

\begin{tabular}{llll}
\hline Risk factors & OR & $\mathbf{9 5 \% ~ C l ~}$ & p-value \\
\hline Age (years) & 17.12 & $3.36-87.12$ & $0.00 I^{*}$ \\
WHR & 0.56 & $0.14-2.17$ & 0.401 \\
GFR $\left(\mathrm{mL} / \mathrm{min} / \mathrm{I} .73 \mathrm{~m}^{2}\right)$ & 0.34 & $0.67-1.73$ & 0.193 \\
Proteinuria & 1.29 & $0.32-5.20$ & 0.720 \\
Kidney transplant status & $1 \mathrm{I} .22$ & $1.82-68.93$ & $0.009^{*}$ \\
Castelli index 2 & 1.95 & $0.57-6.73$ & 0.288 \\
LVH $\left(\mathrm{g} / \mathrm{m}^{2}\right)$ & 2.49 & $0.42-14.60$ & 0.312 \\
\hline
\end{tabular}

Note: *Statistically significant, $p<0.05$.

Abbreviations: WHR, waist-hip ratios; GFR, glomerular filtration rate; LVH, left ventricular hypertrophy.

\section{Discussion}

AsVD was significantly more prevalent among KTRs compared to controls in our study, with nearly half of the KTRs having AsVD. This is comparable with findings from previous studies. ${ }^{17-19}$ Basiratnia et a $1{ }^{17}$ demonstrated a higher mean CIMT in KTRs compared to healthy controls. Similarly, Cader et $\mathrm{al}^{19}$ found a significantly higher prevalence
Table 6 Logistic regression showing relationship of atherosclerotic vascular disease to risk factors among KTRs

\begin{tabular}{llll}
\hline Risk factors & OR & $\mathbf{9 5 \%} \mathbf{C l}$ & p-value \\
\hline Age at transplant ( $>40$ years) & 8.54 & $1.77-41.23$ & $0.008^{*}$ \\
Post-transplant duration ( $>3$ years) & 1.09 & $0.25-4.8 \mathrm{I}$ & 0.914 \\
Duration of dialysis ( $>3$ years) & 1.58 & $0.37-6.80$ & 0.542 \\
$\mathrm{LVH}\left(\mathrm{g} / \mathrm{m}^{2}\right)$ & 2.12 & $0.11-40.53$ & 0.618 \\
\hline
\end{tabular}

Note: *Statistically significant, $p<0.05$.

Abbreviations: KTRs, kidney transplant recipients; LVH, left ventricular hypertrophy.

of increased CIMT among their cohort of KTRs. Although the control group in our study and the study by Basiratnia et $a{ }^{17}$ were similar, in the study by Cader et $a{ }^{19}$ the controls were CKD stage and cardiovascular risk matched. Prevalence of AsVD in our study (46.3\%) is very similar to that reported in a previous study by Japichino et al (46.5\%). ${ }^{18}$ The higher prevalence of AsVD (66.7\%) reported by Cader et $\mathrm{a}^{19}$ could be due to visualization of carotid plaques and measurement of CIMT at the carotid bulb, and also because of differences in participants' profiles with diabetes mellitus accounting for one third of subjects recruited in their study; we had excluded patients with diabetes mellitus and current smokers. The high prevalence of AsVD in our study can be explained by the significant differences in the levels of some established cardiovascular risk factors such as blood pressure, WHR, proteinuria, GFR, LVH, and LAD in KTRs compared to controls.

We found a strong association between AsVD and cardiovascular risk factors. Age, WHR, Castelli indices 1 and 2, non-HDL, AI, LCI, serum fibrinogen levels, LAD, LVH, and mitral valve deceleration time were also significantly associated with the presence of AsVD in our study, comparable to results from previous studies. ${ }^{18-24}$ Kolonko et $\mathrm{al}^{20}$ found age, pre-transplant diabetes, $\mathrm{LVH}$, and CVD to be related to CIMT, which is similar to our findings, despite the exclusion of diabetic KTRs from our study based on the known association between diabetes, dyslipidemia, and atherosclerosis. ${ }^{25}$ Serum fibrinogen has been shown to increase inflammatory and atherosclerotic conditions, possibly through the elaboration of inflammatory cytokines by macrophages involved in atherosclerosis and subsequent stimulation of fibrinogen production by these cytokines. ${ }^{21}$ In our study population, serum fibrinogen was significantly higher among KTRs compared to controls and also significantly higher among participants who had AsVD compared to those without AsVD. AsVD is currently viewed as an inflammatory disease and serum levels of fibrinogen have been influenced by several risk factors of CVD such as hypertension, diabetes, and inflammation..$^{22,23}$ 
Among a cohort of ESKD patients on chronic hemodialysis, serum fibrinogen was found to be higher among patients with fatal and non-fatal cardiovascular events compared to event-free patients. ${ }^{24}$ Our study, which demonstrates an association between AsVD and LVH is supported by reports from an earlier study which described LVH as both a CVD and a risk factor for CVD. ${ }^{26}$

Our study found that age, WHR, Castelli 2, LAD, LVM, and LVMI had positive correlations with CIMT; the association between increased CIMT and age had been described in a previous study. ${ }^{27}$ Also, advancing age has been associated with diminished nitric oxide-mediated vasodilatation and reduction in total nitric production, resulting in endothelial dysfunction. ${ }^{28}$ Increasing age has also been associated with other risk factors for AsVD such as diabetes, hypertension, and vascular calcification. ${ }^{27} \mathrm{LVH}$ is an important predictor of all-cause mortality among KTRs. ${ }^{29}$ Our study showed a negative correlation between LVH and renal function $(r=-0.25$, $p=0.02$ ), consistent with earlier studies in pre-dialysis CKD patients ${ }^{23}$ and KTRs. ${ }^{11}$ An increased WHR has been associated with cardiovascular events among CKD patients; ${ }^{30}$ the finding of a positive correlation between WHR and CIMT in our study is probably due to the use of steroids by the KTRs but may also be explained by the fact that restoration of renal function after kidney transplantation reduces CKDrelated inflammation and malnutrition, enhancing appetite and weight gain.

In our study population, among all the lipid profile and lipoprotein indices analyzed, only Castelli 2 index and LCI correlated positively with CIMT. This finding could be due to the aggressive treatment of dyslipidemia with statins in our patients. Sub-analysis within the KTR group showed that Castelli 2 index retained its positive correlation with CIMT ( $r=0.33 p=0.035)$ while LDL showed a marginally significant correlation. There was no correlation between blood pressure and CIMT, possibly due to the aggressive blood pressure treatment in our KTRs who had a median blood pressure of 139.7/89.5 $\mathrm{mmHg}$.

Age and KTR status were independent predictors of AsVD even after correcting for WHR, proteinuria, GFR, Castelli index 2, and LVH (Table 4). Among KTRs, only age $>40$ years predicted AsVD even when adjusted for $\mathrm{LVH}$, duration of dialysis and post-transplant duration. Increasing age has been demonstrated to be an important predictor of vascular injury ${ }^{28}$ and atherosclerosis has been shown to be associated with changes in CIMT early in the post-kidney transplant period. ${ }^{31}$
This study excluded patients with diabetes mellitus, connective tissue diseases and inflammatory disorders, acute and chronic infections, and smokers. Furthermore, only stable, black KTRs were recruited. This could have contributed to the lower prevalence of AsVD seen in this study compared to the other studies alluded to in the discussion.

In conclusion, AsVD is common among KTRs. Strong correlations exist between CIMT and age, WHR, LVH, LAD, ejection fraction, and mitral valve deceleration time. Among the KTRs' lipoprotein indices, namely Castelli index 2 and LCI, showed a better correlation with CIMT than conventional lipid profile parameters. Age and KTR status were independent predictors of AsVD. The findings of this study suggest that serum fibrinogen, Castelli index 2, and LCI may be important surrogate markers of atherosclerosis in KTRs. It is recommended that the levels of these markers be determined before renal transplantation and monitored in the post-transplant period. In addition, we also recommend more aggressive surveillance for AsVD among KTRs older than 40 years of age.

\section{Acknowledgments}

This work was made possible by the ISN fellowship granted to Dr SO Oguntola to study at the Charlotte Maxeke Johannesburg Academic Hospital, Johannesburg, South Africa, and forms part of his $\mathrm{PhD}$ thesis to be submitted to the University of Witwatersrand. This study was financed through supervisors' (S Naicker and R Duarte) research grants from the National Research Foundation of South Africa.

The authors wish to express their appreciation to Jamie Leigh-Hayes for her assistance during data collection.

\section{Disclosure}

The authors report no conflicts of interest in this work.

\section{References}

1. Schnuelle P, Lorenzo D, Trede M, Van Der Woude FJ. Impact of cadaveric transplantation on survival in end-stage renal failure: evidence for reduced mortality risk compared with hemodialysis during long term follow-up. J Am Soc Nephrol. 1998;9(11):2135-2141.

2. Ribic CM, Holland D, Howell J, et al. Study of cardiovascular outcomes in renal transplantation: a prospective, multicentre study to determine the incidence of cardiovascular events in renal transplant recipients in Ontario, Canada. Can J Kidney Health Dis. 2017;4: 2054358117713729.

3. Ojo AO, Hanson JA, Wolfe RA, et al. Long-term survival in renal transplant recipients with graft function. Kidney Int. 2000;57(1):307-313.

4. Kasiske BL, Guijarro C, Massy ZA, Wiederkehr MR, Ma JZ. Cardiovascular disease after renal transplantation. J Am Soc Nephrol. 1996;7(1):158-165.

5. Vorster HH. The emergence of cardiovascular disease during urbanisation of Africans. Public Health Nutr. 2002;5(1A):239-243. 
6. Peer N, Steyn K, Lombard C, Gaziano T, Levitt N. Alarming rise in prevalence of atherogenic dyslipidaemia in the black population of Cape Town: The Cardiovascular Risk in Black South Africans (CRIBSA) study. Eur J Prev Cardiol. 2014;21(12):1549-1556.

7. Ramjeeth A, Butkow N, Raal FJ, Maholwana-Mokgatihe M. The evaluation of low-density lipoprotein cholesterol goals achieved in patients with established cardiovascular disease and/or hyperlipidaemia receiving lipid-lowering therapy: the South African Not at Goal Study (SA-NAG). Cardiovasc J Afr. 2008;19(2):88-94.

8. Sumner AE, Zhou J, Doumatey A, et al. Low HDL-cholesterol with normal triglyceride levels is the most common lipid pattern in West Africans and African Americans with metabolic syndrome: implication for cardiovascular disease prevention. CVD Prev Control. 2010;5(3): 75-80.

9. Amira OC, Naicker S, Manga P, et al. Adiponectin and atherosclerosis risk factors in African hemodialysis patients: a population at low risk for atherosclerotic cardiovascular disease. Hemodial Int. 2012; 16(1):59-68.

10. Muhammad AS, Naidoo S, Manga P, Nazir MS, Naicker S. Left ventricular hypertrophy in kidney transplant recipients in Sub-Saharan Africa. Sub-Saharan African Journal of Medicine. 2015;2(2):70-73.

11. Kinosian B, Glick H, Garland G. Cholesterol and coronary heart disease: predicting risks by levels and ratios. Ann Intern Med. 1994;121(9): 641-647.

12. Zhu L, Lu Z, Zhu L, et al. Lipoprotein ratios are better than conventional lipid parameters in predicting coronary heart disease in Chinese Han people. Kardiol Pol. 2015;73(10):931-938.

13. Mosteller RD. Simplified calculation of body-surface area. $N$ Engl J Med. 1987;317(17):1098.

14. Cai G, Shi G, Xue S, Lu W. The atherogenic index of plasma is a strong and independent predictor for coronary artery disease in the Chinese Han population. Medicine (Baltimore). 2017;96(37):e8058.

15. Earley A, Miskulin D, Lamb EJ, Levey AS, Uhliq K. Estimating equations for glomerular filtration rate in the era of creatinine standardization: a systematic review. Ann Intern Med. 2012;156(11):785-795.

16. Lang RM, Bierig M, Devereux RB, et al. Recommendations for chamber quantification: a report from the American Society of Echocardiography's Guidelines and Standards Committee and the Chamber Quantification Writing Group, developed in conjunction with the European Association of Echocardiography, a branch of the European Society of Cardiology. J Am Soc Echocardiogr. 2005;18(12):1440-1463.

17. Basiratnia M, Fazel M, Lotfi M, et al. Subclinical atherosclerosis and related risk factors in renal transplant recipients. Pediatr Nephrol. 2010;25(2):343-348.
18. Japichino GG, Bonati L, Rubini P, Capocasale E. [Prevalence of atherosclerosis in renal transplant recipients]. Minerva Cardioangiol. 2001;49(4):229-238. Italian.

19. Cader RA, Zakaria NI, Yaacob Y, Shah SA. Carotid intima-media thickness in kidney transplant recipients. Hong Kong Journal of Nephrology. 2016;19:36-41.

20. Kolonko A, Chudek J, SWzotowska M, Kuczera P, Wiecek A. Cardiovascular risk factors and markers of atherosclerosis in stable kidney transplant recipients. Transplant Proc. 2016;48(5):1543-1550.

21. Koenig W. Fibrin(ogen) in cardiovascular disease: an update. Thromb Haemost. 2003;89(4):601-609.

22. Lee AJ, Lowe GD, Woodward M, Tunstall-Pedoe H. Fibrinogen in relation to personal history of prevalent hypertension, diabetes, stroke, intermittent claudication, coronary heart disease and family history: the Scottish Heart Health Study. Br Heart J. 1993;69(4):338-342.

23. Ridker PM. Inflammation, atherosclerosis and cardiovascular risk: an epidemiologic view. Blood Coagul Fibrinolysis. 1999;10 Suppl 1: S9-S12.

24. Zoccali C, Mallamaci F, Tripepi G, et al. Fibrinogen, mortality and incident cardiovascular complications in end-stage renal failure. J Intern Med. 2003;254(2):132-139.

25. Hong EG, Ohn JH, Lee SJ, et al. Clinical implications of carotid artery intima media thickness assessment on cardiovascular risk stratification in hyperlipidemic Korean adults with diabetes: the ALTO study. BMC Cardiovasc Disord. 2015;15:114.

26. Leigh JA, O’Neal WT, Soliman EZ. Echocardiographic left ventricular hypertrophy as a predictor of cardiovascular disease independent of left ventricular anatomy in persons $\geq 65$ years of age. Am J Cardiol. 2016;117(11):1831-1835

27. Khutan H, Aggarwal S, Kajal KS, et al. Study of carotid intima media thickness in essential hypertension with or without left ventricular hypertrophy. Ann Afr Med. 2017;16(4):192-195.

28. Lyons D, Roy S, Patel M, Benjamin N, Swift CG. Impaired nitric oxidemediated vasodilatation and total body nitric oxide production in healthy old age. Clin Sci (Lond). 1997;93(6):519-525.

29. Paolletti E, Cannella G. Reducing the risk of left ventricular hypertrophy in kidney transplant recipients: the potential role of mammalian target of rapamycin. Transplant Proc. 2009;41(6 Suppl):S3-S5.

30. Elsayed EF, Tighiouart H, Weiner DE, et al. Waist-hip ratio and body mass index as risk factors for cardiovascular events in CKD. Am J Kidney Dis. 2008;52(1):49-57.

31. Nafar M, Khatami F, Kardavani B, et al. Atherosclerosis after kidney transplantation: changes of intima media thickness of carotids during early posttransplant period. Urol J. 2007;4(2):105-110.

International Journal of Nephrology and Renovascular Disease

\section{Publish your work in this journal}

The International Journal of Nephrology and Renovascular Disease is an international, peer-reviewed open access journal focusing on the pathophysiology of the kidney and vascular supply. Epidemiology, screening, diagnosis, and treatment interventions are covered as well as basic science, biochemical and immunological studies. The manuscript
Dovepress

management system is completely online and includes a very quick and fair peer-review system, which is all easy to use. Visit http://www. dovepress.com/testimonials.php to read real quotes from published authors. 